\title{
Cation-diffusion controlled formation of thin graphene oxide composite membranes for efficient ethanol dehydration
}

\author{
Kecheng Guan, Quan Liu, Guanyu Zhou, Guozhen Liu, Yufan Ji, Gongping Liu and Wanqin Jin*
}

\begin{abstract}
Structural manipulation of graphene oxide (GO) building blocks has been widely researched. Concerning GO membranes for separation applications, the validity and maintenance of their microscopic structures in the chemical environment are pivotal for effective separation at the molecular scale. Cationic interactions with both aromatic rings and oxygenated functional groups of GO make metal ions intriguing for physically and chemically structural reinforcement. By filtrating GO suspension through the substrate loaded with cations, stacking of GO nanosheets and diffusion of cations steadily evolve simultaneously in an aqueous environment without flocculation. Thus, thin and homogeneous GO membrane is obtained. Divalent and monovalent cations were studied regarding their interactions with GO, and the performance of correspondingly functionalized membranes was evaluated. The divalent cation-stabilized membranes have favorable stability in the separation of water/ethanol. This facile fabrication and functionalization method may also be applicable for structure construction of other two-dimensional materials.
\end{abstract}

Keywords: graphene oxides, cation diffusion, composite membranes, pervaporation

\section{INTRODUCTION}

Graphene oxide (GO) is a classic type of two-dimensional building blocks [1,2] for the assembling of related composites [3-5]. Generally, researchers in various fields persistently pursue better performances as well as good mechanical strength and chemical adaptability. With the aid of abundant oxygenated functional groups from GO $[6,7]$, subsequent functionalization such as chemical modification is feasible to acquire desirable properties. Although GO nanosheets themselves can be constructed into diversified configurations like laminated and porous structures, the relatively weak interactions (Van der Waals force and hydrogen bonding) between adjacent nanosheets can hardly meet the demand of mechanical strength or chemical stability. In this respect, covalent or non-covalent approaches [8] exerting stronger interactions $[9,10]$ with $\mathrm{sp}^{2}$ cluster and $\mathrm{sp}^{3}$ matrix of GO have been intensively attempted to functionalize GO-based materials. The $\mathrm{sp}^{2}$ cluster is usually attractive for its noncovalent $\pi$-interactions while the $\mathrm{sp}^{3}$ matrix is targeted as active sites where chemical decorations (generally the covalent reaction) occur. These functionalization manners are adjustable before or after the construction of GO-based materials as determined by the properties of the precursor.

The pioneering work on unimpeded water transport through graphene-based membrane [11] has elicited a bunch of interesting experiments [12-14] and simulations [15-17] to study the fast-selective mass transfer through GO-based laminates. From nano-sized particles (e.g., carbon dots [18]), small molecules (e.g., piperazine [19], surfactant $[20,21]$ ) to polymer chains (e.g., polyvinyl alcohol [22]), different chemical species are manipulated to modulate nanostructures and properties of GO-based membranes. With the methodology to functionalize GObased materials, several kinds of membranes for separating specific mixtures [23-30] have been developed. Particularly for liquid separation processes like nanofiltration [31] and pervaporation [32], the structural stability of GO membranes is essential for molecular separation as GO may disintegrate when adsorbing excessive solvent molecules [33,34], and this situation may get worse for the liquid with a high flow rate promoting turbulence across the membrane surface to reduce con-

State Key Laboratory of Materials-Oriented Chemical Engineering, College of Chemical Engineering, Nanjing Tech University, Nanjing 211816, China

* Corresponding author (email: wqjin@njtech.edu.cn) 
centration polarization. The common interactions introduced in GO-based membranes are electrostatic attraction [30,35,36], amino-group involved covalent bonding $[37,38]$ and $\pi-\pi$ stacking [39-42], which are typically sourced from polyelectrolytes, diamine and carbon nanotubes, respectively. By virtue of these modified membrane structures, GO laminates have capability to sustain the high performance during the liquid-related separation process. Using cations to stabilize GO films for mechanical enhancement was substantiated as early as 2008 [43]. Recent studies have reported the cationic interactions with GO to acquire universal coating layer [44], to obtain selective permeation of cations $[10,45,46]$ or to reinforce the stability of the laminar structure [47]. As cations introduced in the interlayer of GO laminates possess different interactions (such as cation- $\pi$ and coordination) with GO in $\mathrm{sp}^{2}$ and $\mathrm{sp}^{3}$ areas, neighboring GO nanosheets become cohesive, and hence the stability as well as the performance of the bulk GO membrane is improved. While direct doping of cations in GO suspension to obtain interacting GO nanosheets will lead to flocculation and precipitates, the derived GO membrane either should be thick enough [48] to withstand the loose structure or was applied in rejection of larger-sized pollutants [49-51] (e.g., proteins). The separation of smallersized molecules remains challenging for these GO membrane configurations.

Membranes are usually fabricated with a two-layer composite structure comprised of a thin selective layer and a porous substrate with good mechanical property. Our previous studies [36,52] have demonstrated the control of GO laminates using lignin-based cation to fabricate substrate-supported thin GO membranes, which are successfully applied for ion sieving and water/alcohol separation. Besides directly decorating GO with cations, herein, flow-directed stacking [53] of cation-interacted GO nanosheets is realized by employing the substrate as the source to continuously release cations. During the pressurized filtration-directed assembly process, the cations loaded on the filter substrate diffuse to upper suspension via concentration gradient, while GO nanosheets are attracted to gradually form laminates on the substrate, further slowing down the diffusion rate for the steady assembly of GO layer (Fig. 1). The cations not only stabilize the GO laminates with higher structural and performance stability in aqueous environment but also strengthen the interfacial combination of GO and substrate. Different kinds of cations were loaded onto the substrate, and their effects on the separation performance of GO membranes were studied.

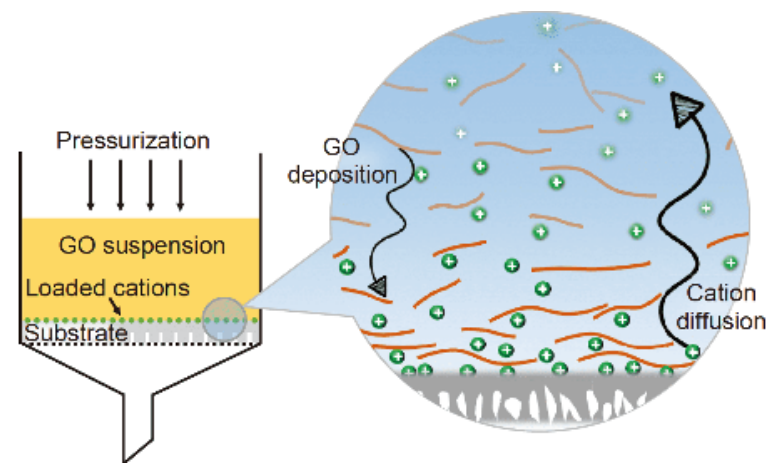

Figure 1 Illustration of filtrating GO aqueous suspension through cations-loaded filter substrate (left) and cation-diffusion controlled GO depositing process (right).

\section{EXPERIMENTAL SECTION}

\section{Preparation of cation-loaded substrate}

Polyacrylonitrile (PAN) ultrafiltration membrane with the average pore size of $20 \mathrm{~nm}$ and thickness of $\sim 60 \mu \mathrm{m}$ (supported by non-woven fabrics, Fig. S1) was selected as the substrate to mechanically support thin GO membrane. PAN substrate was immersed in potassium hydroxide aqueous solution $\left(1.5 \mathrm{~mol} \mathrm{~L}^{-1}\right)$ at $55^{\circ} \mathrm{C}$ for $30 \mathrm{~min}$ to complete the hydrolysis process, turning - $\mathrm{CN}$ group into $-\mathrm{COOH}$. Afterwards, the hydrolyzed PAN (hPAN) substrate was rinsed by ultrapure water (Millipore) several times until the washed water became neutral. The hydrolyzed substrate owns more active sites to load cations.

Different salt solutions $\left(\mathrm{CaCl}_{2}, \mathrm{MgCl}_{2}, \mathrm{NaCl}\right.$ and $\left.\mathrm{LiCl}\right)$ were prepared with the concentration of $0.5 \mathrm{~mol} \mathrm{~L}^{-1}$. hPAN substrates were immersed in these solutions for $24 \mathrm{~h}$ to get different cations loaded. $\mathrm{Ca}^{2+}$ loaded hPAN, for example, is labeled as hPAN-Ca.

\section{Fabrication of thin GO membranes}

GO was synthesized by modified Hummers method [54]. GO aqueous suspension was obtained by dispersing GO flakes in ultrapure water with sonication for $10 \mathrm{~min}$. After that, the as-prepared GO suspension was centrifuged at $12,000 \mathrm{rpm}$ for $10 \mathrm{~min}$, and the supernate was discarded. This process was repeated at another two centrifugation velocities of $10,000 \mathrm{rpm}$ and $8,000 \mathrm{rpm}$ respectively to remove the smaller-sized GO nanosheets. Finally, the GO suspension was filtrated by a nylon filter with pore size of $75 \mu \mathrm{m}$ to remove possible agglomerations.

Determined amount of as-prepared GO suspension was diluted to $200 \mathrm{~mL}$ by water and was followed by filtration 
through the cation-loaded hPAN substrate (effective area of $15 \mathrm{~cm}^{2}$ ) via pressurized filtration process $[55,56]$ under 2 bar to deposit GO nanosheets on the substrate. This process takes around $10 \mathrm{~h}$ to ensure that the water solvent was squeezed out of the membrane as much as possible. At last, the hPAN supported GO composite membrane was dried in a desiccator overnight. Pristine GO membranes without cations were fabricated following the similar procedure above by replacing cation-loaded hPAN with bare PAN.

\section{Evaluation of membrane performance}

The as-prepared membranes were cut into a shape to fit the sealed module. Separation performance was tested via pervaporation process to separate water/ethanol model solution. Detailed apparatus and operations can be found in the previous work [57]. Due to the vacuum state of the permeate side, the permeate is in the form of vapor and is collected with a cold trap in liquid nitrogen. Permeation flux and permeate water content are two indicators to evaluate the separation performance. Gas chromatography analyzes water content (wt\%) of the mixture and the flux $\left(J, \mathrm{~g} \mathrm{~m}^{-2} \mathrm{~h}^{-1}\right)$ is calculated as:

$J=\frac{M}{A t}$,

where $M$ is the mass of collected permeate; $A$ is the effective area of the membrane; $t$ is the operation time.

\section{Calculations of driving force-normalized form of permeation flux and selectivity}

The permeance of individual component $(P / l)_{i}$ (GPU, 1 GPU $=7.501 \times 10^{-12} \mathrm{~m}^{3}$ (STP) $\mathrm{m}^{-2} \mathrm{~s}^{-1} \mathrm{~Pa}^{-1}$ ) and selectivity $(\beta)$ were calculated by the following equations:

$(P / l)_{i}=\frac{J_{i}}{p_{i 0}-p_{i l}}=\frac{J_{i}}{\gamma_{i 0} x_{i 0} p_{i 0}{ }^{\text {sat }}-p_{i l}}$,

$\beta=\frac{(P / l)_{\mathrm{w}}}{(P / l)_{\mathrm{e}}}$,

where $J_{i}\left(\mathrm{~g} \mathrm{~m}^{-2} \mathrm{~h}^{-1}\right)$ is the permeation flux of component $i$; $l(\mathrm{~m})$ is the thickness of membrane; $p_{i 0}$ and $p_{i l}(\mathrm{~Pa})$ are the partial pressures of component $i$ in the feed side and permeate side. $p_{i l}$ can be taken as 0 for the high vacuum situation at the permeate side. $\gamma_{i 0}$ is the activity coefficient of component $i$ in the feed solution; $x_{i 0}$ is the mole fraction of component $i$ in the feed solution; $p_{i 0}^{\text {sat }}(\mathrm{Pa})$ is the saturated vapor pressure of pure component $i$. The permeation flux of water and ethanol should be transformed into the volumes under standard temperature and pressure.

\section{Calculations of activation energy of permeation}

The permeance is dependent on the operating temperature and follows Arrhenius equation:

$P_{i}=P_{0} \mathrm{e}^{-E_{\mathrm{p}} / R T}$,

where $P_{\mathrm{i}}(\mathrm{GPU})$ is the driving force-normalized permeation flux of component $i ; P_{0}$ (GPU) is the pre-exponential factor; $E_{\mathrm{p}}\left(\mathrm{kJ} \mathrm{mol}^{-1}\right)$ is the activation energy; $R$ $\left(\mathrm{kJ} \mathrm{mol}^{-1} \mathrm{~K}^{-1}\right)$ is the gas constant that equals to $8.314 \mathrm{~J} \mathrm{~mol}^{-1} \mathrm{~K}^{-1}$ and $T(\mathrm{~K})$ is the temperature.

Linear dependence can be obtained after taking the log of both sides of Equation (4):

$\ln \left(P_{i}\right)=\ln \left(P_{0}\right)-\frac{E_{\mathrm{p}}}{8.314} \cdot \frac{1000}{T}$.

$E_{\mathrm{p}}$ can be calculated by plotting $\ln \left(P_{i}\right) v s .1000 / \mathrm{T}$.

\section{Density functional theory (DFT) simulation of binding energy}

DFT calculations were performed in CASTEP code to seek a minimum energy structure of cation-stabilized GO. The exchange-correlation interactions were described by the generalized gradient approximation (GGA) in Perdew-Burke-Ernzerhof (PBE) functional $[58,59]$. The cutoff of energy was chosen as $310.0 \mathrm{eV}$ and the selfconsistent field (SCF) tolerance was set as $2 \times 10^{-6} \mathrm{eV} /$ atom. The binding energy $\left(E_{\mathrm{b}}\right)$ between $\mathrm{GO}$ and cation was described by $E_{\mathrm{b}}=E_{\text {total }}-E_{\mathrm{GO}}-E_{\text {cation }}$, where $E_{\text {total }}$ is the total energy of the cation-stabilized GO system; $E_{\mathrm{GO}}$ is the isolated energy of GO and $E_{\text {cation }}$ is the energy of a cation.

\section{Characterizations}

X-ray photoelectron spectroscopy (XPS, Thermo ESCALAB 250, USA) was recorded to reveal the containing elements in different samples. Morphological study and energy dispersive spectroscopy (EDS) of the membrane samples were observed by field emission scanning electron microscope (FESEM, S4800, Hitachi, Japan). The height profiles and roughness parameters of membrane surface were obtained from atomic force microscopy (AFM, Bruker Dimension Icon, Germany) characterizations. X-ray diffraction (XRD, Bruker D8 Advance, Germany) was performed at room temperature with the increment of $0.02^{\circ}$ per second.

\section{RESULTS AND DISCUSSION}

\section{Loading of cations onto substrate}

PAN membrane is an ideal substrate for the stacking of two-dimensional nanosheets owing to its relatively 

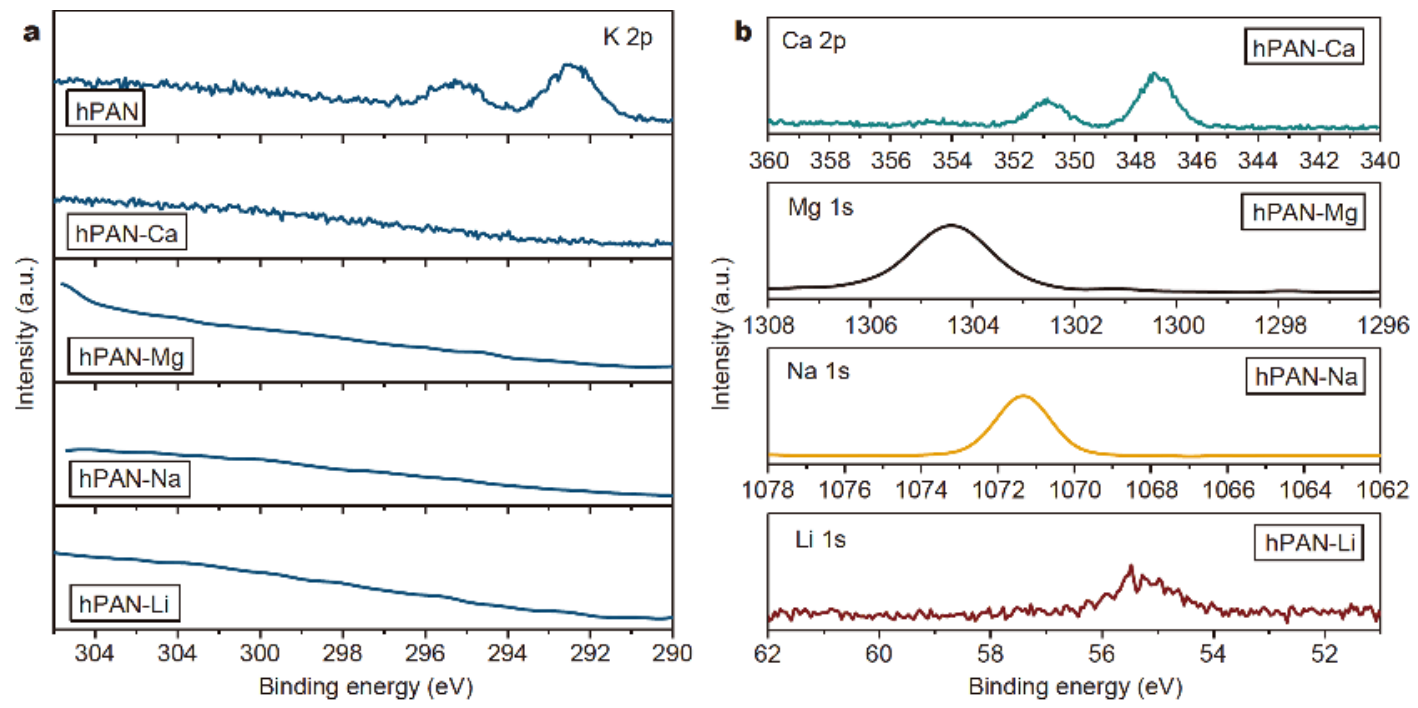

Figure 2 (a) XPS K 2p scan of different hPAN samples; (b) respective narrow scan of different cations-loaded hPAN samples.

smooth surface and comparatively negligible gas or vapor transport resistance. With respect to the loading of cations, after the hydrolysis process, hPAN comprising abundant carboxyl groups (Fig. S2) is more capable of attracting a sufficient amount of cations. As PAN was hydrolyzed by potassium hydroxide, $\mathrm{K}^{+}$could be adsorbed onto the substrate during this process (Fig. 2a). Nevertheless, it was eliminated after the membrane was soaked in a large amount of salt solutions, as evidenced by XPS analysis (Fig. 2a). hPAN substrates loaded with different cations were also revealed by XPS (Fig. $2 b$ and Fig. S3), providing the cation source for the preferential deposition of GO nanosheets.

\section{Cationic interaction and diffusion within GO stacks}

The binding energies between different cations and GO nanosheets were calculated by DFT simulation. The model of GO nanosheet and the original position of cation are the same for all kinds of the cations (Fig. S4). After the system optimization of simulation models, these cations have a divergent tendency to different areas of GO nanosheet. Divalent cations $\left(\mathrm{Ca}^{2+}\right.$ and $\left.\mathrm{Mg}^{2+}\right)$ moved to $\mathrm{sp}^{3}$ area while monovalent cations $\left(\mathrm{Li}^{+}\right.$and $\left.\mathrm{Na}^{+}\right)$kept almost horizontally unchanged (Fig. 3a-d). The calculated binding energy values of these cation-GO systems $\left(\mathrm{Ca}^{2+}\right.$ $\mathrm{GO}, \mathrm{Mg}^{2+}-\mathrm{GO}, \mathrm{Li}^{+}-\mathrm{GO}$ and $\left.\mathrm{Na}^{+}-\mathrm{GO}\right)$ are $-11.54,-13.73$, -3.10 and $-4.14 \mathrm{eV}$, respectively. As a result, divalent cations impart obviously stronger interactions with GO nanosheets, which is helpful to stabilize GO laminates.

Flocculation of GO was observed instantly when divalent cations were added in the suspension as strong in- teractions between them were established, disarranging the dispersibility of GO nanosheets in water. However, for monovalent cations, no immediate change was visible with their addition into GO suspension (Fig. 3e). After these suspensions stood for several hours, slight aggregation of monovalent cation-interacted GO occurred (Fig. S5), implying that interactions were also available between monovalent cations and GO, but the effect was just not as strong as the divalent ones. To fabricate homogeneous GO membrane, hPAN-Ca, as an example, was taken to fabricate $\mathrm{Ca}^{2+}$ diffusion-stabilized $\mathrm{GO}$ membrane $(\mathrm{CaGO})$ to validate the cross-linking effect and diffusion behavior of $\mathrm{Ca}^{2+}$ within $\mathrm{GO}$ laminates during the fabrication process. Besides the common coordination (chelating) phenomenon of $\mathrm{Ca}^{2+}$ with carboxyl groups of GO (Fig. 3f), XPS C 1s spectra of pristine GO and $\mathrm{CaGO}$ membranes were also conducted and it was found that the content of the $\mathrm{C}-\mathrm{O}$ group falls into a considerable decline which can be attributed to the ringopening reaction [43] involved with epoxide groups of GO (Fig. 3g, h). These two interactions contribute to the stabilization of the laminar structure of GO, giving the potential to be applied in the liquid separation process.

Vertical distribution of $\mathrm{Ca}$ element through $\mathrm{CaGO}$ membrane (GO layer thickness of ca. $100 \mathrm{~nm}$ ) was assessed by XPS with detections at different nominal etching depths. The $\mathrm{Ca} / \mathrm{C}$ atomic ratio varies from 0.022 to 0.044 detected from the membrane surface (without etching) to a nominal etching depth of $100 \mathrm{~nm}$, revealing the cation diffusion manner within the GO laminates (Fig. 4). Since GO nanosheets are gradually deposited 
a
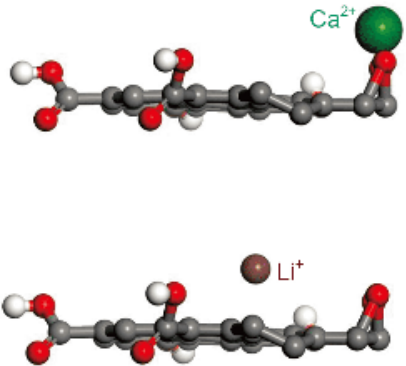

f.

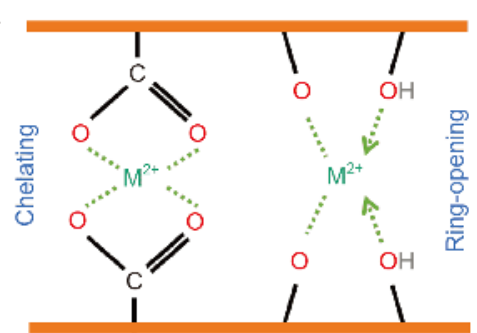

b

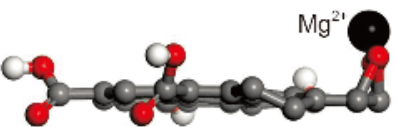

Q $\mathrm{Na}^{\prime}$

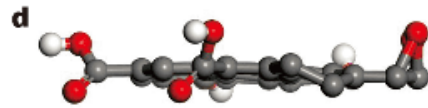

e
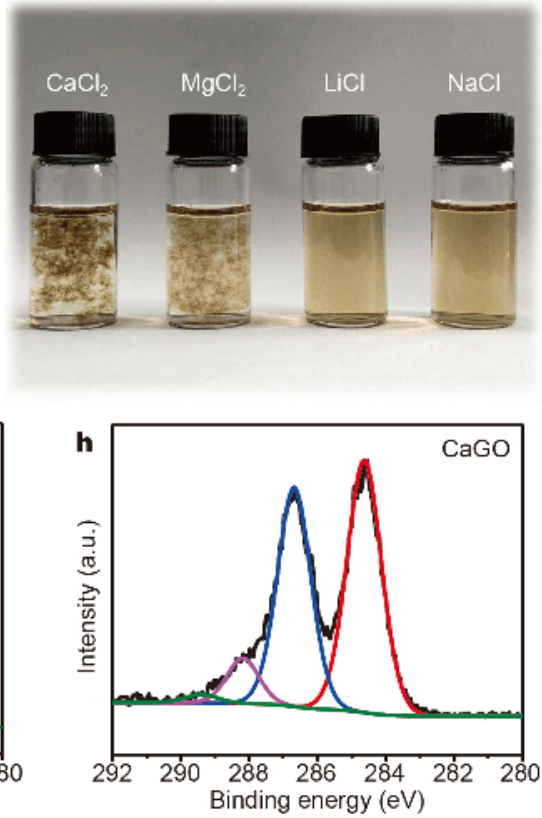

Figure 3 Simulation snapshots of (a) $\mathrm{Ca}^{2+}$, (b) $\mathrm{Mg}^{2+}$, (c) $\mathrm{Li}^{+}$and (d) $\mathrm{Na}^{+}$above GO nanosheet after system optimization; (e) digital image of GO suspensions with different cations added (GO concentration: $0.1 \mathrm{mg} \mathrm{mL}{ }^{-1}$, cation concentration: $2 \mathrm{mmol} \mathrm{L}^{-1}$ ); (f) illustration of interactions between oxygenated functional groups of GO and divalent cation; XPS C 1s spectra of (g) GO and (h) CaGO membranes.
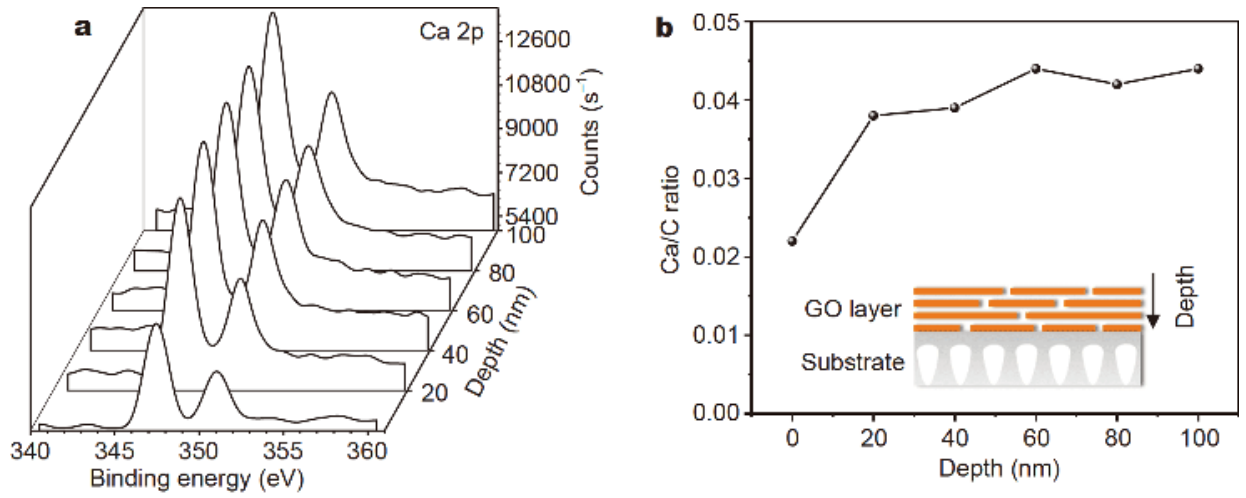

Figure 4 (a) XPS Ca 2p spectra at different etching depths; (b) Ca/C atomic ratio at different etching depths (inset image indicates the depth direction etched from membrane surface).

onto the substrate to form laminar stacks, the diffusion rate of $\mathrm{Ca}^{2+}$ will slow down, therefore preventing the flocculation of GO nanosheets in the suspension and presenting a content gradient of cations throughout the final membrane. Accordingly, accompanied by the cationic interactions, GO deposition steadily evolves until the water solvent is completely filtrated out, and the final stabilized membrane is constructed.

\section{Cation diffusion-derived GO membranes}

With the aid of cation diffusion process, the $\mathrm{Ca}^{2+}$ stabi- lized GO layer was fabricated on the hPAN substrate as observed in Fig. 5a. CaGO membrane shows a thickness of less than $100 \mathrm{~nm}$, and a wrinkled surface morphology (Fig. 5b) arised from the nanosheets stacking during membrane fabrication and the stress releasing during membrane drying. EDS mapping (Fig. 5c) displays the uniform distribution of $\mathrm{C}$ and $\mathrm{Ca}$ elements, corresponding to GO nanosheets and substrate-released cations, respectively. The pristine GO membrane shows a similar layer thickness from SEM image (Fig. S6). Cations play the roles of both binders and spacers inside the inter- 

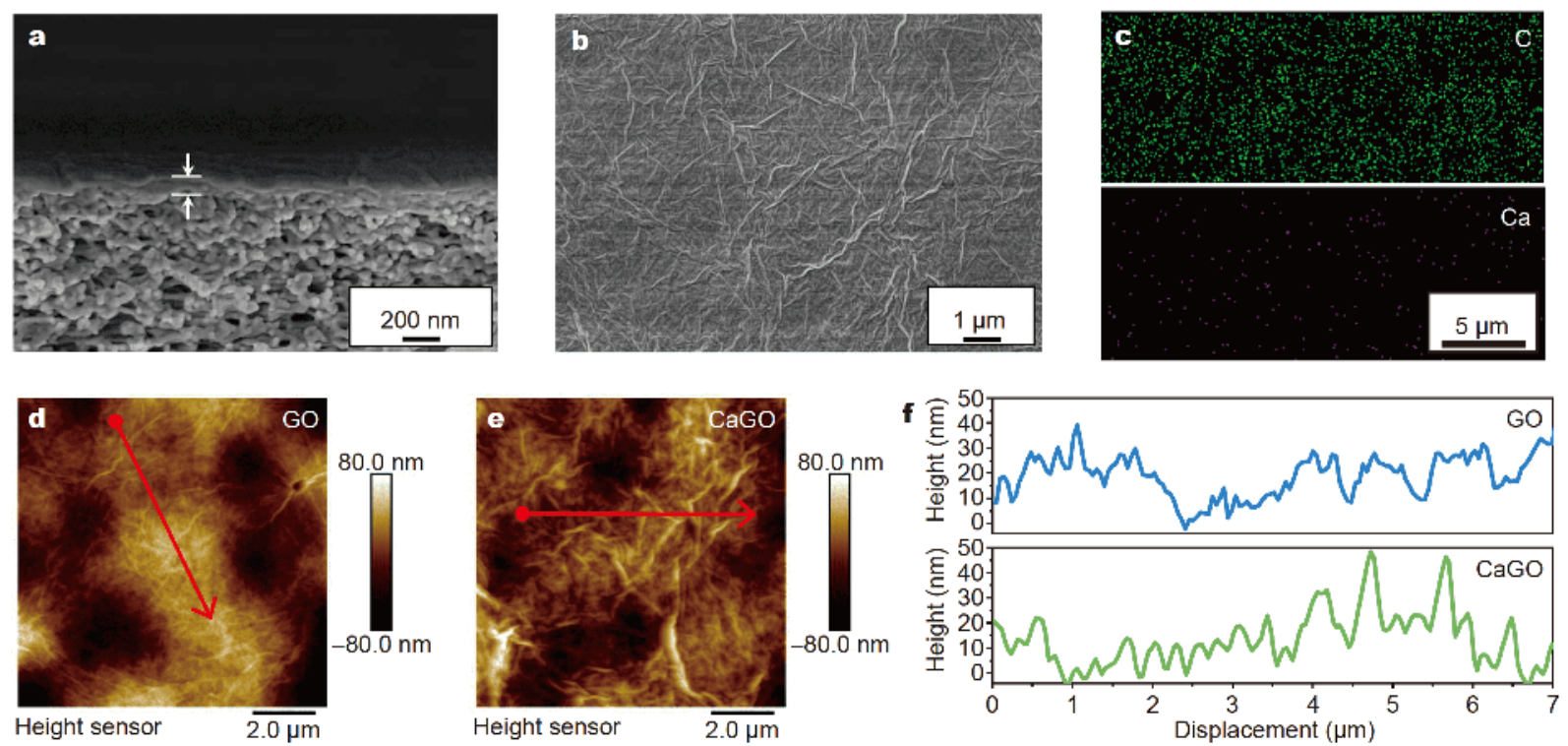

Figure 5 SEM (a) cross-section and (b) surface images of CaGO membrane; EDS mapping of (c) C and Ca element of CaGO membrane; AFM images of (d) GO and (e) CaGO membranes; (f) displacement-height curves of GO and CaGO membrane surfaces (displacement is indicated by red arrows in AFM images).

layers, and therefore the thickness difference between GO and $\mathrm{CaGO}$ could not be the evidence of compaction.

In contrast to the surface morphology of GO membrane, CaGO membrane exhibits more wrinkles arranged across its surface (Fig. S7), probably because of the cation intercalations as well as the interactions between cations and GO nanosheets that alternate the stacking behavior during stacking and drying process. The existence of cations within the GO stacks may also provide the sites for the wrinkle formation. AFM images and relevant height profiles of two different membrane samples also shows the difference of wrinkled pattern (Fig. 5d-f). As suggested by the displacement-height curves in Fig. 5f, CaGO membrane reveals narrower wrinkles than that of GO membrane, which implies a possibility of tightly stacked laminates.

The divalent $\left(\mathrm{Ca}^{2+}, \mathrm{Mg}^{2+}\right)$ and monovalent $\left(\mathrm{Li}^{+}, \mathrm{Na}^{+}\right)$ cations were employed to fabricate correspondingly stabilized GO membranes, designated as $\mathrm{CaGO}, \mathrm{MgGO}$, LiGO and NaGO. Characteristic peaks were observed in all the cation-stabilized GO membranes, indicating the laminar layers of GO membranes (Fig. 6, inset image). The characteristic peaks of monovalent cation-stabilized GO membranes show a slight shift to higher 2-theta value in comparison with pristine GO membrane, suggesting the interaction within the laminates that narrows the interlayer spacing. Although divalent cations can establish much stronger interactions, no apparent shift was found

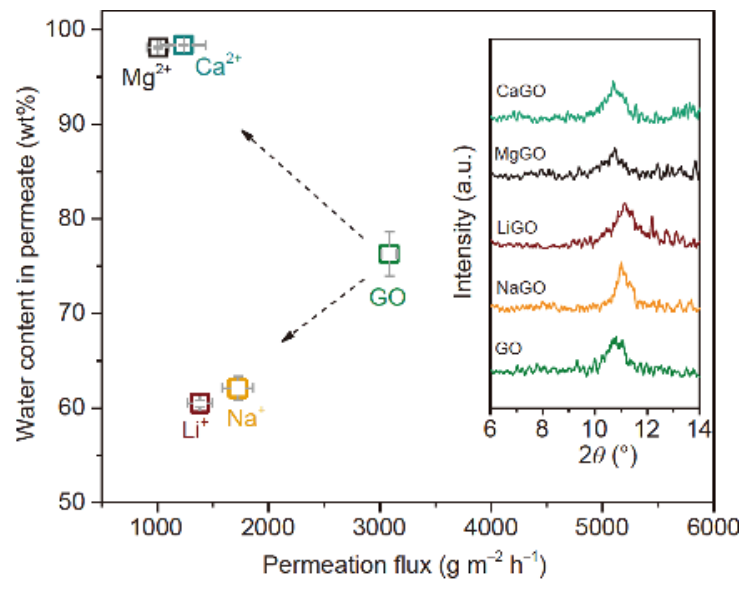

Figure 6 Pervaporation performance of pristine GO membrane and cation-stabilized GO membranes with a feed solution of $10 \mathrm{wt} \%$ water/ ethanol at $70^{\circ} \mathrm{C}$. The inset image is the XRD pattern of corresponding membranes.

in the spectra of divalent cation-stabilized GO membranes. This is possibly due to the enlarged interlayer by larger ionic radii and greater amount of released cations involved in the GO deposition. The calculated interlayer spacing values of cation-stabilized membranes $(0.45-0.47 \mathrm{~nm})$ were similar to the dimension of ethanol molecules, hence the size discrimination of molecules as well as the strong affinity with water in GO interlayers were responsible for the molecular separation.

These membranes went through pervaporation test to 
separate $10 \mathrm{wt} \%$ water/ethanol feed solution at $70^{\circ} \mathrm{C}$ (Fig. 6), and as a result, all the cation-stabilized membranes exhibit lower permeation flux caused by ionic cross-linking effect and the hindering of transport pathways by cations in GO laminates. After the pervaporation test, we characterized these wet membrane samples with XRD (Fig. S8) and found all cations-functionalized membranes underwent higher swelling (peaks shifted to lower 2-theta values) compared with pristine GO membrane, possibly due to the existence of hydrated ions within the GO stacks. Since XRD characterization only revealed the overall situation of interlayer spacing, larger value cannot ensure a greater permeation flux. In addition, the cations here tend to locate at the edge-to-edge slit pores of GO laminates due to coordination with carboxyl groups, which also contributes to the higher transport resistance for molecules. As also indicated by Fig. 6, CaGO and MgGO membranes are much more selective to enrich greater water content in permeates than LiGO and NaGO membranes. Since the cations inside the GO laminates will exert both cationic interactions and ion hydration effects within the interlayer, the intensity of cationic interactions determines the stabilization of GO laminates to prevent the transport channels from excessive expanding in water/ethanol solution. For monovalent cations, comparatively much weaker crosslinking of GO nanosheets and greater hydration of ions in the interlayer of laminates both pose adverse impacts on the structural stability of GO membrane during the selective water permeation process, resulting in non-selective transport channels. Therefore, the interactions between cations and GO are critical for constructing selective transport pathways for small molecules.

Besides the function of stabilizing the GO laminates during the pervaporation tests, cations may also have impacts on the cations-GO system, exerting preferential attraction effects on feed solvent molecules. The interactions between solvent molecules and cations are much weaker than those ones between GO and cations [48], so that the cations will not be carried out from the GO membrane. According to our previous work [36], the cation-GO system preferentially attracted different kinds of molecules within different regions of GO nanosheets. Accordingly, cations also possibly affect the selective transport of molecular mixtures.

\section{Deposition amount and membrane stability}

As the total loading amount of one specific kind of cations on the substrate is fixed (the hydrolysis and cationsloading procedure of PAN substrate were the same for specific kind of cations), we assume that varying the deposition amount of GO will lead to different effects on separation performance. More cations can participate in exerting interactions if the suspension contains less GO nanosheets. While more GO nanosheets are added in the suspension, the amount of cations for diffusion is not adequate to render cationic interactions with the whole laminates. Meanwhile, the cation diffusion behavior is also inhibited if the laminar layer becomes thick enough. Pervaporation results give a coincided trend that when more GO is deposited (larger than $0.2 \mathrm{mg}$ ), the permeation flux unusually rises to a certain degree for the sake of inadequate cationic interactions (Fig. S9).

If the GO content in suspension is down to $0.1 \mathrm{mg}$ (GO-0.1), an unstable change of separation performance was repeatedly observed for pristine GO membrane during the temperature-variation test (Fig. 7a). When the feed temperature reaches $60^{\circ} \mathrm{C}$, permeation flux shows a steep increase and the permeate water content is largely cut down. This is usually the indication of structural collapse, failing in the effective molecular separation among the transport channels. As a contrast, CaGO membrane with $0.1 \mathrm{mg}$ deposition (CaGO-0.1) follows a common variation trend that both permeation flux and permeate water content steadily grows with the elevated temperature (Fig. 7b).

Generally, when GO layer gets thicker on a substrate, it is more likely to be peeled off from the substrate for the poor adhesion and combination with the substrate. Therefore, some methods like polydopamine modification $[60,61]$ are deployed to reinforce the attachment of the GO-based layer to the substrate so as to withstand the washing of fluid flow. Here thicker $\mathrm{GO}$ and $\mathrm{CaGO}$ membranes were fabricated with GO deposition amount of $0.4 \mathrm{mg}$ (labeled as GO-0.4 and CaGO-0.4, respectively). After tested for several hours with a feed flow rate of ca. $280 \mathrm{~mL} \mathrm{~min}^{-1}$ at $70^{\circ} \mathrm{C}$, several parts of pristine $\mathrm{GO}$ layer were washed away with bare substrate surface exposed, while CaGO layer kept attached to the substrate (Fig. 7c). Therefore, the cationic interactions here are also helpful for the combination of the substrate and GO layer.

\section{Effects of the operation condition}

Further evaluation of optimal CaGO membrane $(0.2 \mathrm{mg}$ GO deposition amount, $\mathrm{CaGO}-0.2$ ) was carried out to study the effects of operation temperature and feed water content on the separation performance. Continuous rise of permeation flux and permeate water content were obtained with higher operation temperatures (Fig. 8a). The driving force-normalized form of flux (the per- 

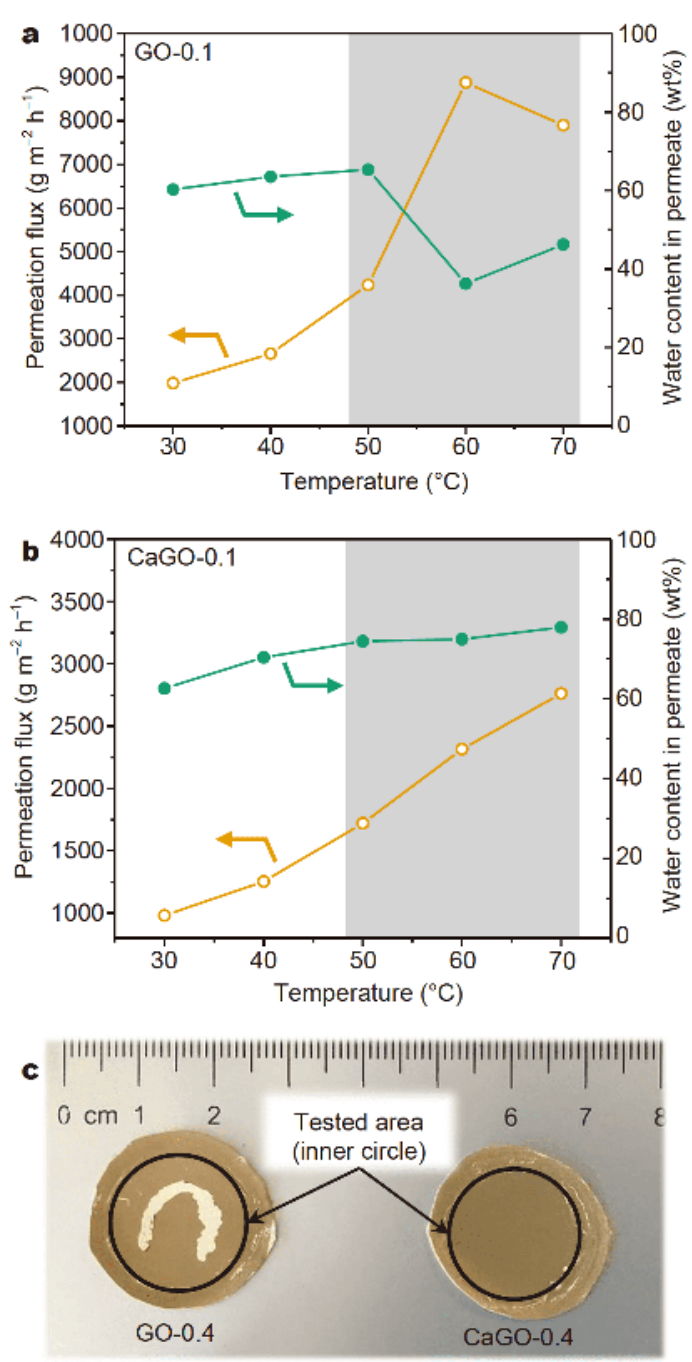

Figure 7 Pervaporation performance of (a) GO-0.1 and (b) CaGO-0.1 membranes at different operation temperatures; (c) digital photo of GO0.4 and CaGO-0.4 membranes after pervaporation test.

meance, see calculations in EXPERIMENTAL SECTION and results in Table S1) also follows the Arrhenius equation with a negative value of activation energy $E_{\mathrm{P}}$ (Fig. S10). As the activation energy of permeation is a combination of activation energy of diffusion $E_{\mathrm{D}}$ and the enthalpy of sorption $\Delta H$, and $E_{\mathrm{D}}$ usually possesses a positive value, so the negative sorption $\Delta H$ dominates the process, leading to the negative value of $E_{\mathrm{p}}$. Although the permeance coefficients decreases under higher temperature, the increase of permeation flux can be attributed that the effect of temperature on saturated vapor pressure is more significant [62].

Feed water content plays the role in acquiring higher separation capacity ranged from around $5 \mathrm{wt} \%$ to $20 \mathrm{wt} \%$. Higher water content in the feed affords greater driving
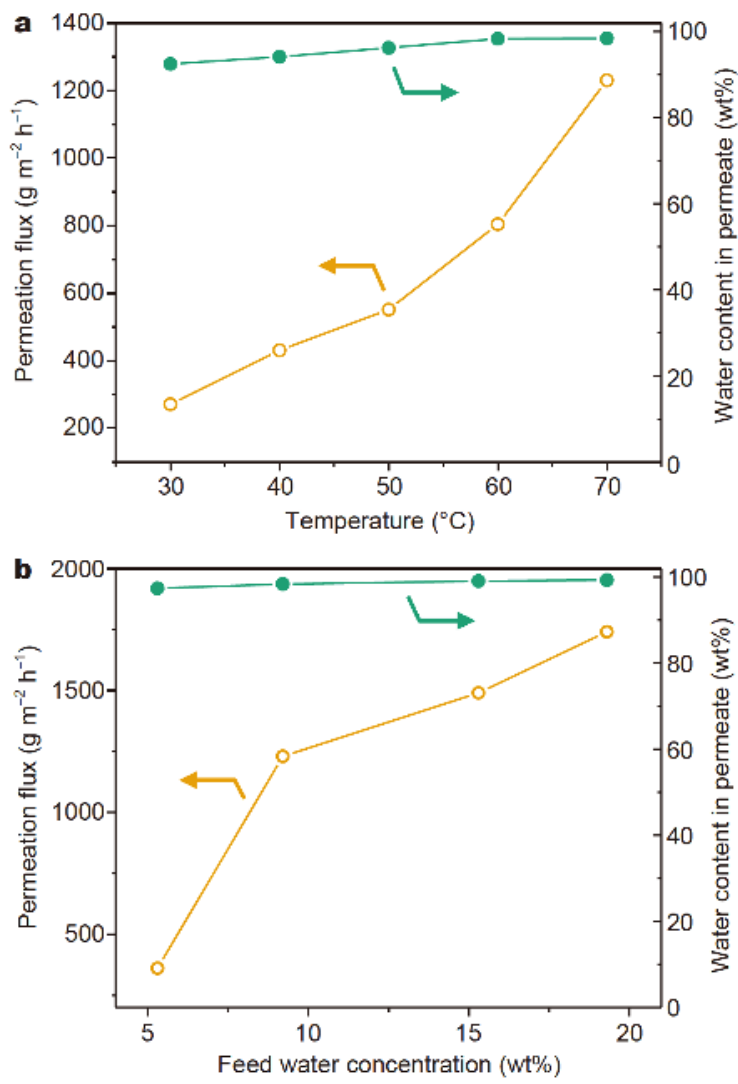

Figure 8 Pervaporation performances (a) under different operation temperatures and (b) with different feed water contents.

force, which is benign for higher permeation flux (Fig. 8b). Meanwhile, the membrane structure may also get swelled at this situation, but with cationic interactions within the laminar structure, CaGO membrane still exhibited excellent selectivity for water/ethanol, reaching $99.0 \mathrm{wt} \%$ water content in permeate when the feed water content is larger than $15 \mathrm{wt} \%$.

Two-stage long-term evaluation of CaGO-0.2 membrane was conducted with a duration of around $100 \mathrm{~h}$ (Fig. 9). The permeation flux values tend to be stable with some reasonable fluctuations both for the first stage of $10 \mathrm{wt} \%$ feed water and the second stage of $20 \mathrm{wt} \%$. The water content in the permeate maintained stably in both periods and is consistent with the variation trend in Fig. 8b. Thus, it indicates that the CaGO membrane is well constructed and the structure is viable for the liquid separation.

\section{CONCLUSIONS}

A simple yet valid method has been demonstrated utilizing cation diffusion to interact with GO nanosheets in aqueous suspension while filtration-directed assembly is 


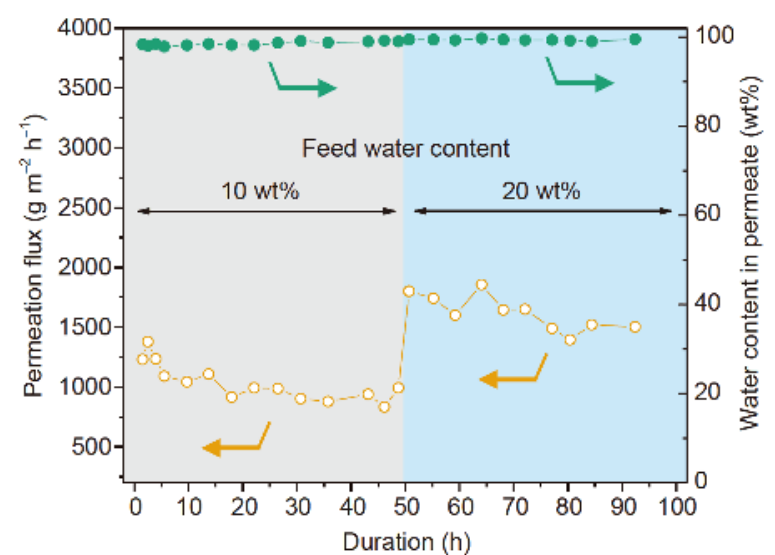

Figure 9 Two-stage long-term pervaporation performance of CaGO membrane.

underway. The cations released from the substrate exert stronger interactions than primitive van der Waals force and hydrogen bond within the laminates to strengthen the structural stability. Divalent cations are proved to be more effective than monovalent ones regarding the effective construction of GO membrane with improved pervaporation performance. Such membrane preserves the stability in variable conditions of feed temperature and feed water content, and the viability of long-term operation is also ensured. This fabrication method of the stabilized membrane may be applicable as well to ameliorate structures constructed by other two-dimensional materials.

Received 12 December 2018; accepted 29 January 2019; published online 26 February 2019

1 Liu G, Jin W, Xu N. Two-dimensional-material membranes: a new family of high-performance separation membranes. Angew Chem Int Ed, 2016, 55: 13384-13397

2 Zhu J, Hou J, Uliana A, et al. The rapid emergence of two-dimensional nanomaterials for high-performance separation membranes. J Mater Chem A, 2018, 6: 3773-3792

3 Huang X, Qi X, Boey F, et al. Graphene-based composites. Chem Soc Rev, 2012, 41: 666-686

4 Huang X, Yin Z, Wu S, et al. Graphene-based materials: synthesis, characterization, properties, and applications. Small, 2011, 7: 18761902

5 Dong Y, Wu ZS, Ren W, et al. Graphene: a promising 2D material for electrochemical energy storage. Sci Bull, 2017, 62: 724-740

6 Zhu Y, Murali S, Cai W, et al. Graphene and graphene oxide: synthesis, properties, and applications. Adv Mater, 2010, 22: 39063924

7 Dreyer DR, Park S, Bielawski CW, et al. The chemistry of graphene oxide. Chem Soc Rev, 2010, 39: 228-240

8 Georgakilas V, Otyepka M, Bourlinos AB, et al. Functionalization of graphene: covalent and non-covalent approaches, derivatives and applications. Chem Rev, 2012, 112: 6156-6214

9 Sun P, Zhu M, Wang K, et al. Selective ion penetration of graphene oxide membranes. ACS Nano, 2013, 7: 428-437

10 Sun $\mathrm{P}$, Zheng F, Zhu M, et al. Selective trans-membrane transport of alkali and alkaline earth cations through graphene oxide membranes based on cation- $\pi$ interactions. ACS Nano, 2014, 8: 850-859

11 Nair RR, Wu HA, Jayaram PN, et al. Unimpeded permeation of water through helium-leak-tight graphene-based membranes. Science, 2012, 335: 442-444

12 Liu G, Jin W, Xu N. Graphene-based membranes. Chem Soc Rev, 2015, 44: 5016-5030

13 Huang H, Ying Y, Peng X. Graphene oxide nanosheet: an emerging star material for novel separation membranes. J Mater Chem A, 2014, 2: 13772-13782

14 Huang L, Zhang M, Li C, et al. Graphene-based membranes for molecular separation. J Phys Chem Lett, 2015, 6: 2806-2815

15 Cohen-Tanugi D, Grossman JC. Water desalination across nanoporous graphene. Nano Lett, 2012, 12: 3602-3608

16 Jiang DE, Cooper VR, Dai S. Porous graphene as the ultimate membrane for gas separation. Nano Lett, 2009, 9: 4019-4024

17 Compton OC, Cranford SW, Putz KW, et al. Tuning the mechanical properties of graphene oxide paper and its associated polymer nanocomposites by controlling cooperative intersheet hydrogen bonding. ACS Nano, 2012, 6: 2008-2019

18 Wang W, Eftekhari E, Zhu G, et al. Graphene oxide membranes with tunable permeability due to embedded carbon dots. Chem Commun, 2014, 50: 13089-13092

19 Zhou F, Tien HN, Xu WL, et al. Ultrathin graphene oxide-based hollow fiber membranes with brush-like $\mathrm{CO}_{2}$-philic agent for highly efficient $\mathrm{CO}_{2}$ capture. Nat Commun, 2017, 8: 2107

20 Lian B, Deng J, Leslie G, et al. Surfactant modified graphene oxide laminates for filtration. Carbon, 2017, 116: 240-245

21 Meng W, Gall E, Ke F, et al. Structure and interaction of graphene oxide-cetyltrimethylammonium bromide complexation. J Phys Chem C, 2015, 119: 21135-21140

22 Morelos-Gomez A, Cruz-Silva R, Muramatsu H, et al. Effective $\mathrm{NaCl}$ and dye rejection of hybrid graphene oxide/graphene layered membranes. Nat Nanotech, 2017, 12: 1083-1088

23 Joshi RK, Carbone P, Wang FC, et al. Precise and ultrafast molecular sieving through graphene oxide membranes. Science, 2014, 343: $752-754$

24 Abraham J, Vasu KS, Williams CD, et al. Tunable sieving of ions using graphene oxide membranes. Nat Nanotech, 2017, 12: 546-550

25 Yang Q, Su Y, Chi C, et al. Ultrathin graphene-based membrane with precise molecular sieving and ultrafast solvent permeation. Nat Mater, 2017, 16: 1198-1202

26 Huang K, Liu G, Lou Y, et al. A graphene oxide membrane with highly selective molecular separation of aqueous organic solution. Angew Chem Int Ed, 2014, 53: 6929-6932

27 Chi C, Wang X, Peng Y, et al. Facile preparation of graphene oxide membranes for gas separation. Chem Mater, 2016, 28: 2921-2927

28 Li H, Song Z, Zhang X, et al. Ultrathin, molecular-sieving graphene oxide membranes for selective hydrogen separation. Science, 2013, 342: 95-98

29 Kim HW, Yoon HW, Yoon SM, et al. Selective gas transport through few-layered graphene and graphene oxide membranes. Science, 2013, 342: 91-95

30 Shen J, Liu G, Huang K, et al. Subnanometer two-dimensional graphene oxide channels for ultrafast gas sieving. ACS Nano, 2016, 10: 3398-3409

31 Zhang Y, Chung TS. Graphene oxide membranes for nanofiltra- 
tion. Curr Opin Chem Eng, 2017, 16: 9-15

32 Huang K, Liu G, Jin W. Vapor transport in graphene oxide laminates and their application in pervaporation. Curr Opin Chem Eng, 2017, 16: 56-64

33 Zheng S, Tu Q, Urban JJ, et al. Swelling of graphene oxide membranes in aqueous solution: characterization of interlayer spacing and insight into water transport mechanisms. ACS Nano, 2017, 11: 6440-6450

34 Aba NFD, Chong JY, Wang B, et al. Graphene oxide membranes on ceramic hollow fibers-Microstructural stability and nanofiltration performance. J Membrane Sci, 2015, 484: 87-94

35 Huang $\mathrm{T}$, Zhang L, Chen $\mathrm{H}$, et al. Sol-gel fabrication of a nonlaminated graphene oxide membrane for oil/water separation. J Mater Chem A, 2015, 3: 19517-19524

36 Guan K, Liu Q, Ji Y, et al. Precisely controlling nanochannels of graphene oxide membranes through lignin-based cation decoration for dehydration of biofuels. ChemSusChem, 2018, 11: 23152320

37 Hung WS, Tsou CH, De Guzman M, et al. Cross-linking with diamine monomers to prepare composite graphene oxide-framework membranes with varying $d$-spacing. Chem Mater, 2014, 26: 2983-2990

38 Shen G, Zhao J, Guan K, et al. Highly efficient recovery of propane by mixed-matrix membrane via embedding functionalized graphene oxide nanosheets into polydimethylsiloxane. AIChE J, 2017, 63: $3501-3510$

39 Goh K, Jiang W, Karahan HE, et al. All-carbon nanoarchitectures as high-performance separation membranes with superior stability. Adv Funct Mater, 2015, 25: 7348-7359

40 Han Y, Jiang Y, Gao C. High-flux graphene oxide nanofiltration membrane intercalated by carbon nanotubes. ACS Appl Mater Interfaces, 2015, 7: 8147-8155

41 Chen X, Qiu M, Ding H, et al. A reduced graphene oxide nanofiltration membrane intercalated by well-dispersed carbon nanotubes for drinking water purification. Nanoscale, 2016, 8: 56965705

$42 \mathrm{Ni} \mathrm{H}, \mathrm{Xu}$ F, Tomsia AP, et al. Robust bioinspired graphene film via $\pi-\pi$ cross-linking. ACS Appl Mater Interfaces, 2017, 9: 2498724992

43 Park S, Lee KS, Bozoklu G, et al. Graphene oxide papers modified by divalent ions-enhancing mechanical properties via chemical cross-linking. ACS Nano, 2008, 2: 572-578

44 Zhao X, Gao W, Yao W, et al. Ion diffusion-directed assembly approach to ultrafast coating of graphene oxide thick multilayers. ACS Nano, 2017, 11: 9663-9670

45 Chen L, Shi G, Shen J, et al. Ion sieving in graphene oxide membranes via cationic control of interlayer spacing. Nature, 2017, 550: $380-383$

46 Cho YH, Kim HW, Lee HD, et al. Water and ion sorption, diffusion, and transport in graphene oxide membranes revisited. J Membrane Sci, 2017, 544: 425-435

47 Yeh CN, Raidongia K, Shao J, et al. On the origin of the stability of graphene oxide membranes in water. Nat Chem, 2015, 7: 166-170

48 Long Y, Wang K, Xiang G, et al. Molecule channels directed by cation-decorated graphene oxide nanosheets and their application as membrane reactors. Adv Mater, 2017, 29: 1606093

49 Liu T, Yang B, Graham N, et al. Trivalent metal cation cross-linked graphene oxide membranes for NOM removal in water treatment. J Membrane Sci, 2017, 542: 31-40

50 Zhang Y, Japip S, Chung TS. Thermally evolved and boron bridged graphene oxide (GO) frameworks constructed on microporous hollow fiber substrates for water and organic matters separation. Carbon, 2017, 123: 193-204

51 Yu W, Yu TY, Graham N. Development of a stable cation modified graphene oxide membrane for water treatment. 2D Mater, 2017, 4: 045006

52 Guan K, Liang F, Zhu H, et al. Incorporating graphene oxide into alginate polymer with a cationic intermediate to strengthen membrane dehydration performance. ACS Appl Mater Interfaces, 2018, 10: 13903-13913

53 Putz KW, Compton OC, Segar C, et al. Evolution of order during vacuum-assisted self-assembly of graphene oxide paper and associated polymer nanocomposites. ACS Nano, 2011, 5: 6601-6609

54 Hummers Jr. WS, Offeman RE. Preparation of graphitic oxide. J Am Chem Soc, 1958, 80: 1339

55 Guan K, Zhao D, Zhang M, et al. 3D nanoporous crystals enabled $2 \mathrm{D}$ channels in graphene membrane with enhanced water purification performance. J Membrane Sci, 2017, 542: 41-51

56 Zhang M, Guan K, Shen J, et al. Nanoparticles@rGO membrane enabling highly enhanced water permeability and structural stability with preserved selectivity. AIChE J, 2017, 63: 5054-5063

57 Xiangli F, Chen Y, Jin W, et al. Polydimethylsiloxane (PDMS)/ ceramic composite membrane with high flux for pervaporation of ethanol-water mixtures. Ind Eng Chem Res, 2007, 46: 2224-2230

58 Perdew JP, Burke K, Ernzerhof M. Generalized gradient approximation made simple. Phys Rev Lett, 1996, 77: 3865-3868

59 Segall MD, Lindan PJD, Probert MJ, et al. First-principles simulation: ideas, illustrations and the CASTEP code. J Phys-Condens Matter, 2002, 14: 2717-2744

60 Liu Q, Wang N, Caro J, et al. Bio-inspired polydopamine: a versatile and powerful platform for covalent synthesis of molecular sieve membranes. J Am Chem Soc, 2013, 135: 17679-17682

61 Yang HC, Waldman RZ, Wu MB, et al. Dopamine: just the right medicine for membranes. Adv Funct Mater, 2018, 28: 1705327201705327

62 Feng X, Huang RYM. Estimation of activation energy for permeation in pervaporation processes. J Membrane Sci, 1996, 118: $127-131$

Acknowledgements This work was financially supported by the National Natural Science Foundation of China (21476107, 21490585, 21776125 and 51861135203), the Innovative Research Team Program by the Ministry of Education of China (IRT17R54) and the Topnotch Academic Programs Project of Jiangsu Higher Education Institutions (TAPP).

Author contributions Jin W had the idea of controlling the assembly of graphene oxide membranes using cation-diffusion. Guan $\mathrm{K}$ and Jin $\mathrm{W}$ designed the experiments. Guan K engineered GO-based membranes and performed the performance evaluation. Liu Q conducted the theoretical simulations. Guan K, Zhou G, Liu G and Ji Y took part in the characterizations of membrane samples. Guan K, Liu G and Jin W analyzed the data and discussed the results. Guan $\mathrm{K}$ wrote the paper with support from Liu G and Jin W.

Conflict of interest The authors declare that they have no conflict of interest.

Supplementary information Supplementary results are available in the online version of the paper. 


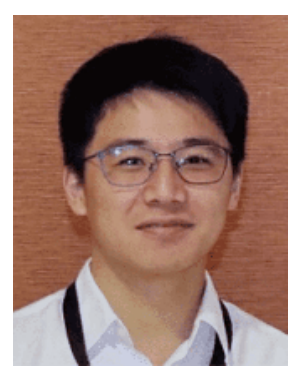

Kecheng Guan is now a PhD candidate at the State Key Laboratory of Materials-oriented Chemical Engineering, Nanjing Tech University. His current research focuses on the development of graphene-based materials for membrane separation processes.

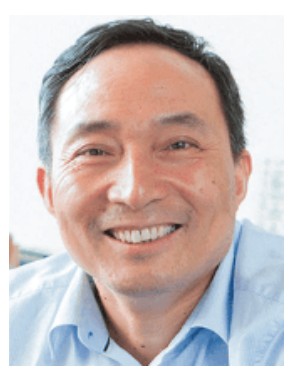

Wanqin Jin is a professor of chemical engineering at Nanjing Tech University, and a Fellow of the Royal Society of Chemistry. He received his PhD degree from Nanjing University of Technology in 1999. He was a research associate at the Institute of Materials Research \& Engineering of Singapore (2001), an Alexander von Humboldt Research Fellow (20012013), and visiting professor at Arizona State University (2007) and Hiroshima University (2011, JSPS invitation fellowship). His current research focuses on membrane materials and processes. He now serves as an Editor of Journal of Membrane Science.

\section{阳离子扩散引导组装的氧化石墨烯膜及其在乙醇脱水中的应用}

管科成, 刘泉, 周冠宇, 刘国振, 季雨凡, 刘公平, 金万勤 ${ }^{*}$

摘要 氧化石墨烯片层组装中的结构调控已有广泛的研究涉及, 对于应用在分离过程的氧化石墨烯薄膜来说, 其微结构在化学环境中的 稳定性和有效性是实现分子分离的一个重要基础. 金属阳离子因其和氧化石墨烯片层上的六元环区域和氧化官能团区域具有相互作用而 能加强氧化石墨烯材料的结构物化性质. 本论文采用负载了金属阳离子的基膜用于抽滤氧化石墨烯分散液沉积成膜, 在此过程中, 水相里 氧化石墨烯片层的堆叠和阳离子的扩散同时发生且不会因为阳离子的引入而导致氧化石墨烯分散液絮凝. 通过这种制备方法, 最终得到 了薄而均匀的分离膜层. 论文研究了二价和一价阳离子对氧化石墨烯膜层形成的影响以及膜性能的变化, 其中二价阳离子修饰后的氧化 石墨烯膜表现出了更为显著的结构稳定性和分离性能. 该简便有效的制备和改性方法对于其他二维材料的结构组装控制也具有一定意义. 